\title{
The Definition and Carrier of Information
}

\author{
Yong Duan \\ College of Engineering, China Agricultural University, Beijing, China
}

Keywords: Information theory, Systems theory, Information, Definition, Symmetry breaking

\begin{abstract}
Claude Elwood Shannon defined the amount of information as the difference between two uncertainties, so information is symmetry breaking. At present, the meaning of information is very confusing. Sometimes it refers to symmetry breaking of consciousness, sometimes it refers to symmetry breaking of signals and symbols, and sometimes it refers to symmetry breaking of substances other than signals and symbols. These three meanings must be strictly distinguished. The concept of information is not scientific, which will bring a lot of troubles and mistakes to scientific research. The word "information" should only indicate the symmetry breaking of matter other than signals and symbols. The reason why signals and symbols can contain information is that organisms can associate; they can associate a signal or symbol with the symmetry breaking of a substance. Signals are substances that can make people and animals associate, and symbols are man-made and easily copied signals.
\end{abstract}

\section{Introduction}

What is information? We all find it difficult to explain clearly. Even Norbert Wiener, the founder of Cybernetics, had no choice but to say, "information is information, neither matter nor energy." Shannon, the founder of Information Theory, did not give the definition of information directly. In the mathematical description of information, he defined the amount of information as the difference between two uncertainties, which implied his definition of information: information is something we used to eliminate uncertainty. In Shannon's information theory, uncertainty is expressed and measured by probability entropy (information entropy). Therefore, Shannon's definition of information can also be expressed as, information is the matter that we used to reduce entropy. The amount of information is the reduction of entropy, so information is negative entropy.

\section{Information is the Symmetry Breaking of Matter}

Wiener said, "the concept of information amount is very naturally subordinate to entropy, a classical concept of statistical mechanics. Just as the amount of information in a system is a measure of its degree of organization, the entropy of a system is a measure of its degree of disorganization; this one is exactly the negative number of that one.” [1] According to Boltzmann's principle, entropy is directly proportional to the logarithm of the number of complexion. The state with large number of complexion is the equilibrium state; the state with little number of complexion, or negative entropy corresponds to the symmetry breaking of structure [2]. If information is negative entropy, then information is symmetry breaking. Symmetry breaking is the difference of structure, so the essence of information is the difference of structure. Various functions of information in biological and non-living systems are the decisive role of structure on function.

There are two kinds of symmetry breaking: symmetry breaking of matter (substance) and symmetry breaking of consciousness. When I don't know what's in the glass, I will think that there might be a fish, or a frog, or solution in the glass. When I believe that there is a fish in the glass, I deny the possibility that there is something else in the glass. This is the symmetry breaking of consciousness. The symmetry breaking of consciousness is not the information defined by Wiener.

There are two kinds of symmetry breaking of matter: one is the symmetry breaking of signals and symbols, or signifier; the other is the symmetry breaking of substances other than signals and symbols, or signified. The specific structure of each system, especially the specific structure of a 
complex system, is a kind of symmetry breaking. Signals and symbols are some special substances; their specific structures are also symmetry breaking. For example, there is a fish in the glass, and the specific structure of the fish is a kind of symmetry breaking. If I put a sign with "fish" on it next to the glass, this word is a symbol. The symbol is also a kind of symmetry breaking. Because I wrote "fish”, it means I didn't write "horse”, “cow”, “sheep” or "solution”. The symmetry breaking of symbols is not the information defined by Wiener.

\section{Information is Not Scientific Language}

At present, the meaning of information is very confusing. Sometimes it refers to the symmetry breaking of consciousness. De Gao said, "information is knowledge”. Sometimes it refers to the symmetry breaking of signals and symbols; "information is signal, data, and intelligence". Sometimes it refers to the symmetry breaking of materials other than signals and symbols, which means "the uneven distribution of matter and energy in time and space, and the difference or variation of things."[3] These three meanings must be strictly distinguished because they have completely different attributes. The structure of DNA is a material structure, not a symbol. The basic group symbol we write on paper is a symbol, rather than the actual structure of the substance it represents. Symbols on the paper cannot directly participate in chemical reactions and guide the synthesis of proteins, while the base pairs in DNA can guide the synthesis of proteins. We often say that "computers process information". In fact, computers can not deal with the actual structure of DNA and other substances; it can only process their symbols. A computer program written on paper is a symbol of symmetry broken, but the computer cannot understand this program. The order of electrical signals generated by tapping on the keyboard is the symmetry breaking of matter, which is the information that computer can recognize.

If a person has two names, there is no problem. For example, if I say "Zedong Mao" and "Runzhi Mao", you will know what I mean and there will be no misunderstanding. However, if two people share the same name, it is very troublesome and easy to cause misunderstanding. For example, if Bang Liu has two subordinates called Xin Han, it will be easy to misunderstand without explanation. Therefore, people's names are often not scientific languages. In the household registration management system, the state has set an ID card number for everyone. This number is not allowed to be repeated and will not cause any misunderstanding, so the ID card number is a scientific language. Scientific language is a language that cannot be misunderstood.

Now the term information is not a scientific name, because it has three meanings, and is easy to be misunderstood. The concept of information is not scientific, which will bring a lot of troubles and mistakes to scientific research. The word "fish" has different attributes from the actual fish. Fish can be eaten, but the word can't be eaten. If information is used to explain the nature of life, the evolution of the universe or any other theory, huge troubles will appear. Jianhui Li believes that the essence of life is the transmission of information in the process of growth and reproduction. [4] What he said refers to the symmetry breaking of matter other than signals and symbols, but others may understand it as symmetry breaking of signals and symbols, or symmetry breaking of consciousness.

Information defined by entropy refers to the structure of matter, rather than symbols or consciousness. However, it has not been noticed in the past. There is a serious misunderstanding. In the study of information sources and channels, information refers to symbols, rather than structure of matters. But Wiener and other scholars have never made any explanation about this; maybe they cannot make a clear distinction themselves.

How to solve this problem? Different nouns must be used to denote these three kinds of symmetry breaking. I advocate that we should not use the word information to express the symmetry breaking of consciousness or the symmetry breaking of signals and symbols. We should only use the word information to express the symmetry breaking of matters other than signals and symbols. The matter mentioned here is opposite to consciousness.

The symmetry breaking of signals and symbols should be called as signals and symbols. It can be said that signals and symbols are carriers of information, but signals and symbols themselves are 
not information. The breaking of symmetry of consciousness is called the cognition of information. Cognition and other consciousness are not information. Now we are used to calling signals as information, so in order to avoid misunderstanding, I will try not to use the word "information", and always use "structure" instead of "information". It does not mean that the word of "information" can't be used; but the premise is that it doesn't cause misunderstanding, or it doesn't matter if it causes misunderstanding.

\section{Carrier of Information}

When we talk about information, many people regard the carrier of information as information. The footprints of dinosaurs are the carrier of information; the existence and characteristics of dinosaurs are information. The same information carrier can transmit different information. For the same pit on the rock, if it is dug by a man, it cannot transmit information of dinosaurs. The glass windows broken by artificial blasting and smashed by children's football also transmit different messages. So it is totally wrong to regard the carrier of information as information.

The carrier of information is not information, so whether the information is included in the information carrier? It can be said that, but we must make it clear that the exact meaning of such inclusion is completely different from that in general sense. Does the rock pit "include" dinosaurs? The pit is so small and the dinosaur is so big; of course the pit doesn't include dinosaurs.

Signals and symbols themselves are not information, but why do we always say that signals and symbols contain information? In Pavlov's conditioned reflex experiment, the bell did not contain information on food. Even if you say to the dog, "ringing the bell means feeding", the dog can't understand the information in the bell. But after the bell and the food appeared at the same time many times, the information contained in the bell was recognized by the dog. The bell is a signal. The appearance of bell and food is a kind of material symmetry breaking. Why the dog can recognize or process this information? This is because dogs have association established in their nervous systems. The reason that signals and symbols can contain information is that organisms can associate; they can associate a signal or symbol with the symmetry breaking of substance. Therefore, signals are substances that can make people and animals associate, and symbols are man-made and easily copied signals. Signals include man-made and non-man-made substances that are easy to replicate and hard to replicate. Symbol is a special signal.

Peirce once gave a definition of symbol. He said: "I define the symbol as something A, which brings something B (i.e., its explanation term) into something C (its object) corresponding to it. In this way, A itself (the symbol itself) replaces C." This definition is inaccurate because "not all symbols have logical explanatory terms." And this definition does not point out the difference between signals and symbols. "I think the essential function of symbols is to make inefficient signifier relationships more effective," Pierce said. [5] But pierce did not explain reasons for the increase in efficiency.

Without biological association, signals and symbols cannot contain information; there are only objective matters and their interaction. For example, primitive humans used rattan to tie a knot to indicate that they had caught a sheep today. This rattan knot contains information; but in the objective world, it has nothing to do with sheep. A vine ties a knot in the process of natural growth, can this knot represent a sheep? It has nothing to do with sheep even if there are sheep eating grass near it every day. If it does not interact with consciousness, rattan knot will never become the carrier of information. In the same way, a sheep can be represented by knots, by a certain distribution of ink, or by several electrical pulses. In the objective world, rattan knots, ink and electric pulse have nothing in common. Only when they interact with consciousness can they have something in common, that is, they can remind people of a sheep.

Saussure hoped to establish the discipline of semiotics. "We can imagine a science that studies symbolic life in a social context; it's part of social psychology and a part of general psychology; we call it semiotics," he said. "Semiotics will tell us what constitutes symbols and what rules govern them. Linguistics is only a part of general semiotics. If I have succeeded in finding a place for Linguistics in various sciences, it is because I have linked it with semiotics."[6] But Saussure failed 
to establish semiotics. The semiotics he said is actually the Information Theory and philosophy of language, not psychology. However, today, Information Theory and philosophy of language do not provide accurate definitions of information, symbol and language, and do not analyze the role of language from the perspective of generalized evolution.

When people see anything, they may have a lot of different associations. Common language is the agreement of associative contents. Language is a symbol; daily language, computer language, telegraph code, password and so on are all agreed common languages.

A lot of consciousness and information do not interact with each other before the formation of consciousness. After consciousness came into being, their roles changed. There have been electromagnetic waves in the universe for a long time, but if they don't interact with consciousness, they can only be electromagnetic waves, and will never be the carrier of information. The so-called interaction with consciousness is the discovery of this kind of electromagnetic wave and the emergence of a new understanding, such as the identification of a supernova.

The process of decoding is a cognitive process. The signal is the output of the objective material system. In decoding, the objective material system is regarded as a black box, and the structure model of the system is established based on the output of the black box.

Decoding requires two things: the first is information carrier; the second is logic. Information carrier cannot contain logic, let alone logical models. The logical model is established by people's association and other conscious activities. People often think that this model is an imitation of a certain prototype, so they go to search the prototype. Where is the prototype? If we regard the maker of information carrier (such as dinosaurs) as the prototype, there is a problem. People have not seen dinosaurs, how can they imitate them? What people can see is the information carrier, so they think that the prototype is hidden in the information carrier. This ideological process is exactly the same as Plato's, which is objective idealism. People can build models with or without prototypes. People can create a model with their own imagination, and people can create the image of dinosaurs without seeing living dinosaurs. When people believe that these models are objective, they seem to see the prototype. Computers can depict dinosaurs vividly. In fact, the so-called prototype is just a model. Only when we see dinosaurs tens of millions of years ago, can we say that we have seen the prototype.

The unit of information is bit, but how much information is one bit? The criteria are not objective, but subjective. Information is the structure of matter; objective matter is infinitely complex. Therefore, any object, even a small nail, has infinite information with countless bits. But only a small amount of information is effective. If people can react differently to different information, then the information is effective. No matter how large the amount of invalid information is, if people can't distinguish them, they will be treated equally. Therefore, bit is the number of types of human reaction. If a person can make a response to a change of information, it means that a person has received 1 bit of information.

\section{Conclusion}

At present, the meaning of information is not unified, which brings a lot of troubles and mistakes to scientific research and our daily communication. Through analysis, this paper holds that the word "information" should only indicate the symmetry breaking of matters other than signals and symbols. At the same time, we must pay attention to the difference between information and information carrier.

\section{References}

[1] Wiener, N. Cybernetics (Science of Control and Communication in Animals and Machines), 2nd edition, Beijing: Science Press, 1963, pp. 11.

[2] Duan, Y. Reconsideration of Boltzmann Formula and the Second Law of Thermodynamics. WOP in Engineering and Science Research, vol.14, no.4, pp.3-7, 2019. 
[3] Gao, D. Theory of Order: the Essence of Xiangshu and Philosophy of Traditional Chinese Medicine, Hohhot: Inner Mongolia People's Publishing House, 2002, pp. 13-14.

[4] Li, J.H. Friendship with Truth: Philosophical Reflections on Modern Science, Shanghai: Shanghai Science and Technology Education Press, 2002, pp. 37.

[5] Peirce, C.S. On Signs, Chengdu: Sichuan University Press, 2014, pp. 31-48.

[6] Saussure, F. Course in General Linguistics, Beijing: China Social Sciences Press, 2009, pp. 19. 